\title{
Envisioning security control in renewable dominated power systems through stochastic multi-period AC security constrained optimal power flow
}

\author{
Mohammad Iman Alizadeh, Muhammad Usman, Florin Capitanescu \\ Environmental Research and Innovation (ERIN) Department, Luxembourg Institute of Science and Technology (LIST) \\ Belvaux, Luxembourg \\ mohammad.alizadeh@list.lu, muhammad.usman@list.lu, florin.capitanescu@list.lu
}

\begin{abstract}
The accelerated penetration rate of renewable energy sources (RES) brings environmental benefits at the expense of increasing operation cost and undermining the satisfaction of the N-1 security criterion. To address the latter issue, this paper envisions N-1 security control in RES dominated power systems through stochastic multi-period AC security constrained optimal power flow (SCOPF). The paper extends the state-ofthe-art, i.e. deterministic and single time period AC SCOPF, to capture two new dimensions, RES stochasticity and multiple time periods, as well as emerging sources of flexibility such as flexible loads (FL) and energy storage systems (ESS). Accordingly, the paper proposes and solves for the first time a new problem formulation in the form of stochastic multi-period AC SCOPF (S-MP-SCOPF). The S-MP-SCOPF is formulated as a non-linear programming (NLP) problem. It computes optimal setpoints of flexibility resources and other conventional control means for congestion management and voltage control in day-ahead operation. Another salient feature of this paper is the comprehensive and accurate modelling, using: AC power flow model for both pre-contingency and post-contingency states, intertemporal constraints for resources such as FL and ESS in a 24hours time horizon and RES uncertainties. The importance and performances of the proposed model through a direct approach, pushing the problem size up to the solver limit, are illustrated on two test systems of 5 nodes and 60 nodes, respectively, while future work will develop a tractable algorithm.

Index Terms-congestion management, energy storage systems, flexibility, flexible loads, security-constrained optimal power flow, voltage control
\end{abstract}

\section{NOMENCLATURE}

\section{Sets}

$E \quad$ Set of ESS indexed by $e$

$F \quad$ Set of FL indexed by $f$

$G \quad$ Set of generators indexed by $g$

$K \quad$ Set of operation states, including normal and contingencies, indexed by $k$

$N \quad$ Set of nodes indexed by $n$

$R \quad$ Subset of nodes with RES

$S \quad$ Set of scenarios indexed by $s$

$T \quad$ Set of time periods indexed by $t$

This research work has received funding from the European Union's Horizon 2020 research and innovation program under grant agreement No 864298 (project ATTEST).

\section{Parameters}

$\Delta P_{G_{g}}$ ramp rate limit of generator $g$

$\Delta T \quad$ time interval ratio

$\eta^{\text {ch,e }} \quad$ charging efficiency rate of ESS $e$

$\eta^{d i s, e}$ discharging efficiency rate of ESS $e$

$\bar{P}_{e}^{c h} / \bar{P}_{e}^{d i s}$ maximum active power charging/discharging limit of storage $e$

$\bar{P}_{f}^{i n c} / \bar{P}_{f}^{d e c}$ maximum active power increase/decrease limit of FL $f$

$\pi_{s} \quad$ probability of occurrence of scenario $s$

$\underline{P}_{g} / \bar{P}_{g}$ minimum/maximum active power limit of generator $g$

$\underline{Q}_{g} / \bar{Q}_{g}$ minimum/maximum reactive power limit of generator ${ }_{g} g$

$\underline{V}_{n} / \bar{V}_{n}$ minimum/maximum voltage limit at node $n$

$B_{n m}^{s h} \quad$ shunt susceptance of the branch linking nodes $n$ and $m$

$B_{n m} \quad$ susceptance of the branch linking nodes $n$ and $m$

$c_{e} \quad$ cost (€/MWh) of active power of energy storage $e$

$c_{f} \quad \operatorname{cost}(€ / \mathrm{MWh})$ of active power of flexible load $f$

$c_{g} \quad$ cost $(€ / \mathrm{MWh})$ of active power re-dispatch of

generator $g$

$c_{n}^{\text {Gcurt }}$ cost (€/MWh) of active power curtailment at node $n$

$c_{n}^{\text {Lcurt }}$ cost $(€ / \mathrm{MWh})$ of active load curtailment at node $n$

$G_{n m} \quad$ conductance of the branch linking nodes $n$ and $m$

$I_{n m}^{\max } \quad$ maximum current of line linking nodes $n$ and $m$

$P_{D_{n, t}} \quad$ load active power at node $n$, period $t$

$P_{G_{g, t}}^{*} \quad$ active power of generator $g$ at time $t$ cleared in the energy market

$Q_{D_{n, t}}$ load reactive power at node $n$, period $t$

$R_{n, s, t}$ RES active power at node $n$, period $t$, scenario $s$

$S O C_{e}^{\max }$ maximum State-of-Charge for storage $e$

$S O C_{e}^{\min }$ minimum State-of-Charge for storage $e$

Variables

$e_{n, s, t}^{k} \quad$ real part of complex voltage $\left(e_{n, s, t}^{k}+j f_{n, s, t}^{k}\right)$ at node $n$, period $t$, scenario $s$, state $k$

$f_{n, s, t}^{k} \quad$ imaginary part of complex voltage at node $n$, period $t$, scenario $s$, state $k$

$L c_{n, s, t}^{k}$ active load curtailment at node $n$, time $t$, scenario $s$, 
state $k$

$P_{e, s, t}^{c h, k} \quad$ active power charging of storage $e$ at time $t$, scenario $s$, state $k$

$P_{e, s, t}^{d i s, k}$ active power discharging of storage $e$ at time $t$, scenario $s$, state $k$

$P_{f, s, t}^{d e c, k}$ active power decrease of FL $f$ at time $t$, scenario $s$, state $k$

$P_{f, s, t}^{i n c, k}$ active power increase of FL $f$ at time $t$, scenario $s$, state $k$

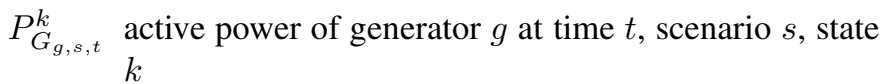

$P_{i n j_{n, s, t}}^{k}$ active power injection at node $n$, time $t$, scenario $s$, state $k$

$Q_{c_{n, s, t}}$ reactive load curtailment at node $n$, time $t$, scenario $s$, state $k$

$Q_{G_{g, s, t}}^{k}$ reactive power of generator $g$ at time $t$, scenario $s$, state $k$

$Q_{i n j_{n, s, t}}^{k}$ reactive power injection at node $n$, time $t$, scenario $s$, state $k$

$R c_{n, s, t}^{k}$ active power of RES curtailment at node $n$, time $t$, scenario $s$ state $k$

$S O C_{e, s, t}^{k}$ State-of-Charge for storage $e$ at period $t$, scenario $s$, state $k$

\section{INTRODUCTION}

\section{A. Motivation}

To attain the stringent sustainable goals set to them, power systems worldwide are hosting increasingly large amounts of renewable energy sources (RES) at all voltage levels. However, massive RES penetration significantly challenges the enforcement of transmission system security [1] due to the inherent variability and difficulty to predict RES output. In this context, power systems operate closer to their security limits and hence fulfilling $\mathrm{N}-1$ security becomes a challenging task, particularly under stressed operation conditions, unexpected RES output, and/or unavailability of effective control actions. Regarding the latter aspect, as classical control means (e.g. conventional power plants) and controllable RES may not be sufficient to fulfill security, additional emerging sources of flexibility, such as flexible loads (FL) and energy storage systems (ESS), are being deployed to enhance power system flexibility and offset issues provoked by RES [2], [3].

Deterministic AC security-constrained optimal power flow (SCOPF) [4]-[6] is the conventional tool to enforce $\mathrm{N}-1$ security at a given period of time. SCOPF is mainly used in the day-ahead operation for the cost-optimal procurement of ancillary services (e.g. for managing congestion and voltages). To this end, SCOPF computes the optimal balance of preventive (i.e. pre-contingency) and corrective (i.e. post-contingency) actions able to guarantee static system security (i.e. pertaining to congestion and voltage magnitude) for a set of postulated (e.g. N-1) contingencies.

A variety of SCOPF problems have been tackled, that target optimizing the redispatch of either active or reactive powers [4], [7]. SCOPF problems are in their simplest form formulated as large scale (non-convex) nonlinear programs (NLPs), whose main difficulty is the large size [4]-[6].

\section{B. Related Works}

Deterministic single period AC SCOPF is state-of-the-art [8]-[15]. Its solution has been extensively explored through various algorithms: decomposition methods (e.g. Benders decomposition or iterative algorithms based on contingencies filtering, both embedding interior-point method for core NLP problem) applied to exact formulations [8]-[11], approximations [9], [12], meta-heuristics [13], and even convex relaxations (e.g. semi-definite programming [8], [14] and second order cone programming [15]) that are able to assess the optimality gap of exact algorithms' solution. Further modeling advancement regarding generators' response after contingencies to frequency and voltage control have been also explored [12], [16].

Solving deterministic single period AC SCOPF is today computationally demanding but still scalable to systems of reasonably large size (i.e. thousand nodes) [6]. Despite AC SCOPF is state-of-the-art, some works develop sophisticated algorithms for its linear (DC) SCOPF approximation via column and constraint generation [17], constraints redundancy screening [18], alternating direction method of multipliers $(\mathrm{ADMM})$ in a distributed manner [19], network compression [20], or machine learning [21].

To capture RES inherent variability, two timely extensions of SCOPF have been developed independently to address:

- uncertainties (regarding RES) based on robust optimization [22], distributionally robust optimization [23], stochastic optimization (exact [24], simplified DC [25] or relaxed [26]), and chance-constrained optimization [27]; other uncertainties (e.g. regarding corrective control potential failure) were tackled via chance-constraints [28].

- multiple time periods (linked by inter-temporal constraints) via the simplified DC model [29], [30] or the exact AC model [31], [32].

However, these extensions are very scarce and tremendously increase the computational burden of the problem.

Additionally, to reliably deal with RES variability, a meaningful SCOPF problem should also consider time dependent emerging flexibility resources (e.g. FL and ESS). However, these flexibility resources were considered only sporadically and in a single period deterministic SCOPF [33].

\section{Paper Contributions and Organization}

It can be concluded that the approaches aimed to extend SCOPF state-of-the-art are not only very scarce but also have considered separately the two main features: RES uncertainties and multiple time periods. In addition, the approaches to any of these two challenges do not model the two other difficult features as AC network model and emerging flexibility resources in a joint fashion.

To bridge this gap, as a conceptual contribution, this paper proposes the new envisioned concept of multi-period AC 
SCOPF under uncertainties to control N-1 security in RESdominated power systems of the future. The main contribution of this paper is the extension of the state-of-the-art, i.e. deterministic AC SCOPF, to capture jointly two new dimensions (RES stochasticity and multiple time periods) as well as the emerging sources of flexibility (FL and ESS). In other words, the paper proposes and solves for the first time a new problem formulation in the form of a stochastic multi-period AC SCOPF (S-MP-SCOPF).

A direct approach relying on the state-of-the-art NLP solver IPOPT [34], widely used in many AC OPF/SCOPF applications, is conducted formulating the largest problem size that the solver can still manage while a tractable algorithm is planned for future work. Note that, the "size challenge" of the proposed S-MP-SCOPF problem is determined by the product of four different dimensions: the size of the system, number of postulated contingencies, number of uncertainty scenarios, and number of time periods.

To further highlight the above mentioned novel contributions of this work, Table I summarizes the main modelling features of the proposed approach which distinguishes it from the several existing methods. One can observe that, like this work, scalability is not pursued per se in most works that address more challenging AS SCOPF problem extensions. Also, it is implied that if AC grid model is not used, then simplified models (e.g. DC) are adopted.

TABLE I

MODEL FEATURES OF VARIOUS APPROACHES

\begin{tabular}{|c|c|c|c|c|c|c|}
\hline model & $\begin{array}{l}\text { deterministic } \\
\text { single-period }\end{array}$ & $\begin{array}{c}\text { multiple } \\
\text { time periods }\end{array}$ & $\begin{array}{c}\text { operation } \\
\text { uncertainty }\end{array}$ & $\begin{array}{l}\text { flexibility } \\
\text { resources }\end{array}$ & $\begin{array}{c}\mathrm{AC} \\
\text { model }\end{array}$ & $\begin{array}{l}\text { scala- } \\
\text { bility }\end{array}$ \\
\hline$|8|-\mid 17$ & $\bar{x}$ & & & & $\bar{x}$ & $\bar{x}$ \\
\hline [18], [21] & $x$ & & & & & $x$ \\
\hline [19] & $x$ & & & & & \\
\hline 22 & & & $x$ & & $x$ & \\
\hline 23 & & & $x$ & & & $x$ \\
\hline 24 & & $x$ & & $x$ & & \\
\hline [25] & & & $x$ & & & $x$ \\
\hline 26 & & & $x$ & & $x$ & \\
\hline |27] & & & $x$ & & $x$ & \\
\hline 28 & & & $x$ & & $x$ & \\
\hline 29 & & $x$ & $x$ & & & $x$ \\
\hline [30] & & $x$ & $x$ & $x$ & & $x$ \\
\hline [31 & & $x$ & & & & \\
\hline [32] & & $x$ & & & $x$ & \\
\hline proposed & & $x$ & $x$ & $x$ & $x$ & \\
\hline
\end{tabular}

The remaining of the paper is organized as follows. Section II presents the detailed formulation of the S-MP-SCOPF problem. Section III provides quantitative results with a direct approach to the proposed problem. Section IV concludes and provide directions for future works.

\section{FORMULATION OF THE S-MP-SCOPF PROBLEM}

This section describes in detail the proposed S-MP-SCOPF model to procure, in day-ahead operation planning, flexibility for congestion management and voltage control such that to satisfy N-1 security criterion. The model computes optimal setpoints for flexibility resources (FL and ESS), RES curtailment, and other conventional control means (e.g. generators)

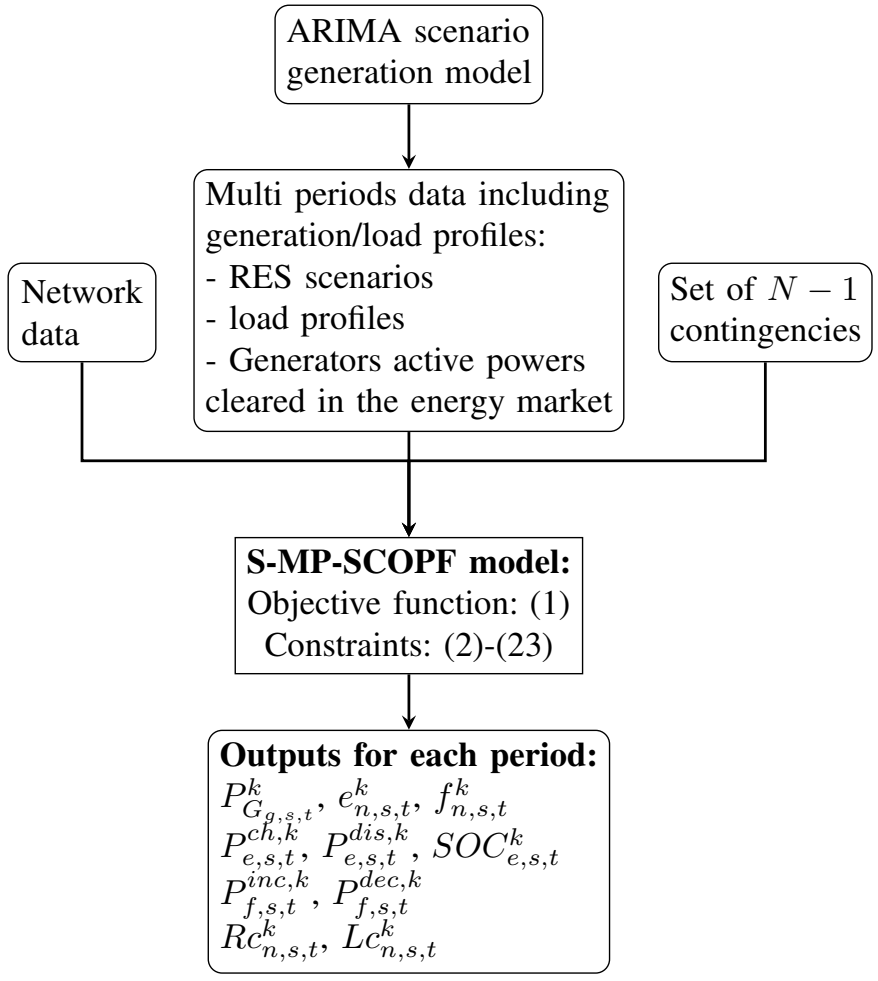

Fig. 1. A flowchart of the proposed model.

in each time period and system state, as illustrated in Fig. 11 The model relies on $\mathrm{AC}$ power flow equations expressed using voltages in rectangular coordinates.

The objective (1) of the S-MP-SCOPF is to minimize the expected cost of flexibility procurement for ancillary services (congestion and voltage control) in transmission network operation under both normal and post contingency states. This cost pertains to the re-dispatch of conventional generators, ESS, and FL, curtailment of RES, and load curtailment to prevent infeasibility.

$$
\begin{aligned}
& \min \sum_{s \in S} \sum_{t \in T} \pi_{s}\left\{\sum_{g \in G}\left(P_{G_{g, s, t}}^{0}-P_{G_{g, t}}^{*}\right) c_{g}\right. \\
& +\sum_{k \in K}\left[\sum_{e \in E}\left(P_{e, s, t}^{c h, k}+P_{e, s, t}^{d i s, k}\right) c_{e}\right. \\
& +\sum_{f \in F}\left(P_{f, s, t}^{i n c, k}+P_{f, s, t}^{d e c, k}\right) c_{f} \\
& \left.\left.+\sum_{n \in R}\left(R c_{n, s, t}^{k}\right) c_{n}^{\text {Gcurt }}+\sum_{n \in N}\left(L c_{n, s, t}^{k}\right) c_{n}^{\text {Lcurt }}\right]\right\}
\end{aligned}
$$


The problem is subject to the following constraints:

$$
\begin{aligned}
& \sum_{g \in G} P_{G_{g, s, t}}^{k}+R_{n, s, t}+\sum_{e \in E}\left(P_{e, s, t}^{d i s, k}-P_{e, s, t}^{c h, k}\right) \\
& +\sum_{f \in F}\left(P_{f, s, t}^{d e c, k}-P_{f, s, t}^{i n c, k}\right)-R c_{n, s, t}^{k}+L c_{n, s, t}^{k}= \\
& P_{D_{n, t}}+P_{i n j_{n, s, t}}^{k} \\
& \forall n \in N, s \in S, t \in T, k \in K
\end{aligned}
$$

$$
\begin{aligned}
& \sum_{g \in G} Q_{G_{g, s, t}}^{k}=Q_{D_{n, t}}-Q_{c_{n, s, t}}+Q_{i n j_{n, s, t}}^{k} \\
& \forall n \in N, s \in S, t \in T, k \in K \\
& P_{i n j_{n, s, t}}^{k}=\left[\left(e_{n, s, t}^{k}\right)^{2}+\left(f_{n, s, t}^{k}\right)^{2}\right] \sum_{m \in N} G_{n m} \\
& -\sum_{m \in N}\left[\left(e_{n, s, t}^{k} e_{m, s, t}^{k}+f_{n, s, t}^{k} f_{m, s, t}^{k}\right) G_{n m}\right. \\
& \left.+\left(f_{n, s, t}^{k} e_{m, s, t}^{k}-e_{n, s, t}^{k} f_{m, s, t}^{k}\right) B_{n m}\right] \\
& \forall n \in N, s \in S, t \in T, k \in K
\end{aligned}
$$$$
Q_{i n j_{n, s, t}}^{k}=-\left[\left(e_{n, s, t}^{k}\right)^{2}+\left(f_{n, s, t}^{k}\right)^{2}\right] \sum_{m \in N}\left(B_{n m}^{s h}+B_{n m}\right)
$$$$
+\sum_{m \in N}\left[\left(e_{n, s, t}^{k} e_{m, s, t}^{k}+f_{n, s, t}^{k} f_{m, s, t}^{k}\right) B_{n m}\right.
$$$$
\left.-\left(f_{n, s, t}^{k} e_{m, s, t}^{k}-e_{n, s, t}^{k} f_{m, s, t}^{k}\right) G_{n m}\right]
$$

$\forall n \in N, s \in S, t \in T, k \in K$

$\underline{P}_{g} \leq P_{G_{g, s, t}}^{k} \leq \bar{P}_{g} \quad \forall g \in G, s \in S, t \in T, k \in K$

$\underline{Q}_{g} \leq Q_{G_{g, s, t}}^{k} \leq \bar{Q}_{g} \quad \forall g \in G, s \in S, t \in T, k \in K$

$\left(G_{n m}^{2}+B_{n m}^{2}\right)\left[\left(e_{n, s, t}^{k}-e_{m, s, t}^{k}\right)^{2}+\left(f_{n, s, t}^{k}-f_{m, s, t}^{k}\right)^{2}\right]$

$$
\leq\left(I_{n m}^{\max }\right)^{2} \quad \forall n, m \in N, s \in S, t \in T, k \in K
$$

$\left(\underline{V}_{n}\right)^{2} \leq\left(e_{n, s, t}^{k}\right)^{2}+\left(f_{n, s, t}^{k}\right)^{2} \leq\left(\bar{V}_{n}\right)^{2}$

$$
\forall n \in N, s \in S, t \in T, k \in K
$$

$\left|P_{G_{g, s, t-1}}^{0}-P_{G_{g, s, t}}^{0}\right| \leq \Delta P_{G_{g}} \quad \forall g \in G, s \in S, t \in T$

$\left|P_{G_{g, s, t}}^{k}-P_{G_{g, s, t}}^{0}\right| \leq \Delta P_{G_{g}}$

$$
\forall g \in G, s \in S, t \in T, k \in K, k \neq 0
$$

$S O C_{e, s, t+1}^{k}=S O C_{e, s, t}^{k}+\Delta T\left(\eta^{c h, e} P_{e, s, t}^{c h, k}-P_{e, s, t}^{d i s, k} / \eta^{d i s, e}\right)$

$\forall e \in E, s \in S, t \in T, k \in K$

$S O C_{e}^{\text {min }} \leq S O C_{e, s, t}^{k} \leq S O C_{e}^{\text {max }}$

$\forall e \in E, s \in S, t \in T, k \in K$

$S O C_{e, s, T}^{k}=S O C_{e, s, 0}^{k}, \forall e \in E, s \in S, t \in T, k \in K$

$0 \leq P_{e, s, t}^{c h, k} \leq \bar{P}_{e}^{c h}, \forall e \in E, s \in S, t \in T, k \in K$

$0 \leq P_{e, s, t}^{d i s, k} \leq \bar{P}_{e}^{d i s}, \forall e \in E, s \in S, t \in T, k \in K$

$\frac{P_{e, s, t}^{c h, k}}{\bar{P}_{e}^{c h}}+\frac{P_{e, s, t}^{d i s, k}}{\bar{P}_{e}^{d i s}} \leq 1, \forall e \in E, s \in S, t \in T, k \in K$

$\sum_{t \in T} P_{f, s, t}^{i n c, k}=\sum_{t \in T} P_{f, s, t}^{d e c, k}, \forall f \in F, s \in S, t \in T, k \in K$

$$
\begin{aligned}
& 0 \leq P_{f, s, t}^{i n c, k} \leq \bar{P}_{f}^{i n c}, \forall f \in F, s \in S, t \in T, k \in K \\
& 0 \leq P_{f, s, t}^{d e c} \leq \bar{P}_{f}^{d e c}, \forall f \in F, s \in S, t \in T, k \in K \\
& \frac{P_{f, s, t}^{d e c, k}}{\bar{P}_{f}^{d e c}}+\frac{P_{f, s, t}^{i n c, k}}{\bar{P}_{f}^{i n c}} \leq 1, \forall f \in F, s \in S, t \in T, k \in K \\
& 0 \leq L c_{n, s, t}^{k} \leq P_{D_{n, t}}, \forall n \in N, s \in S, t \in T, k \in K \\
& 0 \leq R c_{n, s, t}^{k} \leq R_{n, s, t}, \forall n \in N, s \in S, t \in T, k \in K
\end{aligned}
$$

where $k=0$ represents normal operation state while $k \geq 1$ indicates contingency states, all notations being defined in the nomenclature.

Constraints (2) and (3) represent active and reactive power balance equations (for each node $n$, scenario $s$, time $t$ and state $k$ ), which include active/reactive power flows from Eqs (4) and (5). Note that in (3) load curtailment assumes constant power factor.

Constraints (6) and (7) are the hard physical limits on active and reactive powers of generator $g$.

Network operation constraints (congestion and voltages) are modeled by constraints (8) and (9). Eq (8) represents the longitudinal branch current limit, which is a reasonable approximation of the current aimed to avoid doubling the number of constraints (e.g. when the current is expressed for both ends of the branch). Eq (9) imposes limits on node voltage magnitude.

$\mathrm{Eq}(10)$ restricts the ramping of generator $g$ for each two successive time intervals of normal operating state. Eq (11) is the coupling constraint on active power of generator $g$ between normal operation and post-contingency states.

The ESS operation is captured by the following set of constraints [35]. Eq (12) describes the dynamics of Stateof-Charge (SoC), (13) is the SoC limit for each ESS, (14) maintains the the SoC of ESS equal on first and last time periods, Eqs (15) and (16) are limits on active power charging and discharging of ESS period, and (17) prevents simultaneous charging and discharging of storage $e$ for each period.

It is important to note that (17) is a smart and tractable exact approximation, proposed in [36] to avoid introducing binary variables to model the statuses charging and discharging of an ESS. In the latter work it is demonstrated that this modeling matches accurately the effect of using binary variables, i.e. at the optimum an ESS either charges or discharges but not both. This is due to the fact that both charging and discharging statuses have associated costs in the objective function which in turn prevents the simultaneous charging and discharging of an ESS. This effect is also empirically observed in all our numerical simulations.

The FL operation is modeled by the following set of constraints. Eq (18) maintains the energy balance of a FL over whole time horizon, (19) and (20) are the limits on the increase and decrease of active power of FL, respectively, and Eq (21) prevents simultaneous increase and decrease in the active power of FL during each time interval. Remark that (21) relies on the same type of assumption and approximation as for storage elements in Eq (17). 


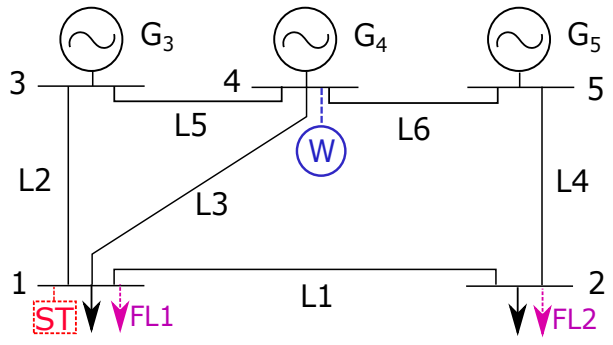

Fig. 2. One-line diagram of the 5-bus system

Finally, (22) limits the load curtailment while (23) restricts the RES curtailment.

Note that the proposed S-MP-SCOPF is an NLP problem.

Last but not least, a modelling aspect worth discussing for any stochastic optimization problem is the number of decisionmaking stages assumed and interpretation/implementation of the optimal stochastic solution. Often, in day-ahead operation planning, there are two such stages corresponding to "here and now" decisions and "wait and see" decisions. While two stages can be straightforwardly modeled, we opted only for modelling all decisions as "wait and see" (i.e. scenario-dependent, as opposed to "here and now" decisions which are scenario independent) for the sake of computational challenge, as it leads to a larger stochastic optimisation challenge. In such case, the transmission system operator can either implement in practice the optimal solution corresponding to one of the assumed scenarios or a weighted (e.g. via probabilities of occurrence) of solutions of all postulated scenarios.

\section{NumeriCAl RESUlTS}

The features of the proposed S-MP-SCOPF model are illustrated using two test systems of 5 and 60 nodes respectively, for 24-hours time frame (one hour time resolution), given sets of $\mathrm{N}-1$ contingencies and different number of scenarios.

All simulations are performed in Julia/JuMP open source programming language [37] on a $\mathrm{PC}$ of $2.11 \mathrm{GHz}$ and $48 \mathrm{~GB}$ of RAM. IPOPT optimizer is used to solve all NLP problems [34].

\section{A. Results for 5-node test system}

The 5 node system is adapted from [6] and its one-line diagram is shown in Fig. 2 Tables $\Pi$ and $\amalg$ respectively show the steady-state and line data for the 5-node test system [6]. We consider $6 \mathrm{~N}-1$ line contingencies and up to 10 uncertainty scenarios. Full results for this test case are comprehensively discussed and all necessary data are provided to enable benchmarking, reproducibility and comparison.

To consider RES, a wind farm is deployed at node 4. Table IV shows ten normalized scenario profiles over 24-hour period which are generated using a time series based Auto regressive integrated moving average (ARIMA) model [38]. The scenarios and contingencies are equiprobable and (for simplicity) loads are assumed constant for the entire 24 hour horizon.
Four case studies are developed to assess the capability of the proposed model (in all cases load and RES generation curtailment is allowed to prevent infeasible problems):

- Case\#0: no FL or ESS are considered;

- Case\#1: one ESS is embedded at node 1 with the parameters provided in Table $\nabla$ and $c_{e}$ cost is set to 80 $€ / \mathrm{MWh}$;

- Case\#2: 10\% of load at node 1 and 2 (FL1 and FL2 in Fig. 2) is assumed flexible and the $c_{f}$ cost is set to 80 and $40 € / \mathrm{MWh}$, respectively in all operation states;

- Case\#3: both ESS and FL are allowed to take part in optimization, with the costs given above.

1) Case\#0: Table VI compares the results of the proposed model for different RES capacities, where RC0-RC10, CG, LC stand for RES capacity (between 0 and 1,000 MW), conventional generation and load curtailment respectively. It can be observed that, as the penetration rate of RES increases, CG cost reduces gradually since the RES production is paid by feed-in-tariff. However, the cost of curtailed energy increases up to $105,294 €$ in RC10. This suggests that efficient utilization of flexibility resources can potentially reduce the amount of curtailed energy.

2) Case\#1: Table VII provides the proposed model results with ESS at node 1 for different RES capacities. In comparison with the base case (i.e. Case\#0) the curtailment cost is reduced up to $46 \%$ (i.e. $(105,294-56,754) / 105,294 €$ ) and the total cost reduces by $1,631,997-1,595,907=36,090 €$ for $\mathrm{RC} 10$. In addition, the flexibility added by ESS prevents load curtailment in case RC7.

3) Case\#2: Similar benefits are observed using FL in both nodes 1 and 2 as shown in Table VIII Using the flexibility provided by FL causes no energy curtailment for RC7 and RC8. Even in the case RC10, the total curtailment cost is reduced to $62 \%$ (i.e. $(105,294-40,001) / 105,294 €)$. In addition, the total cost for $\mathrm{RC} 10$ is reduced by $2.75 \%$ with respect to the base case.

4) Case\#3: The results for the proposed S-MP-SCOPF model considering both ESS and FL are summarized in Table IX It can be seen that no energy curtailment occurs for RC7 and RC9. In addition, the curtailment cost decreases by $90.5 \%$ (i.e. $(105,294-10,001) / 105,294 €)$. One can also observe that the total cost is reduced by $4 \%$ with respect to the base case.

Another important remark is the synergy benefit of simultaneously using FL and ESS flexibility sources as can be noticed by the reduced cost of FL $(9.88 \%)$ and ESS $(27.2 \%)$ in comparison to the results reported for the Case\#1 and Case\#2 in Tables VII and VIII respectively.

The computation time of the NLP problem is short in the range of few tens of seconds. Despite the small system size (5 nodes), the corresponding S-MP-SCOPF problem includes 7 operation states, 24 time periods and 10 scenarios is roughly equivalent in size to solving an $\mathrm{AC}$ OPF problem for a system of cca. 8,400 nodes.

For all case studies, the only binding contingency is the disconnection of line L2. For this contingency, in Case\#3 and 
TABLE II

5-BUS SYSTEM DATA AT PEAK LOAD WITH NO WIND, NO STORAGE AND NO FLEXIBLE LOAD

\begin{tabular}{|c|c|c|c|c|c|c|c|c|c|c|c|c|c|c|c|}
\hline bus & $\begin{array}{l}P_{L} \\
\text { MW }\end{array}$ & $\begin{array}{c}Q_{L} \\
\text { MVar }\end{array}$ & $\begin{array}{c}P_{G} \\
\mathrm{MW}\end{array}$ & $\begin{array}{l}Q_{G} \\
\text { MVar }\end{array}$ & $\begin{array}{l}V \\
\mathrm{pu}\end{array}$ & $\begin{array}{c}V^{\min } \\
\mathrm{pu}\end{array}$ & $\begin{array}{c}V^{\max } \\
\mathrm{pu}\end{array}$ & $\begin{array}{l}P_{G}^{\min } \\
\mathrm{MW}\end{array}$ & $\begin{array}{l}P_{G}^{\max } \\
\mathrm{MW}\end{array}$ & $\begin{array}{l}Q_{G}^{\min } \\
\text { MVar }\end{array}$ & $\begin{array}{l}Q_{G}^{\max } \\
\text { MVar }\end{array}$ & $\begin{array}{l}\Delta P_{G} \\
\mathrm{MW}\end{array}$ & $\begin{array}{c}a \\
€ / \mathrm{MWh}^{2}\end{array}$ & $\begin{array}{c}b \\
€ / M W h\end{array}$ & $\begin{array}{l}c \\
€\end{array}$ \\
\hline 1 & 1100 & 400 & - & - & 0.954 & 0.92 & 1.05 & - & - & - & - & - & - & - & - \\
\hline 2 & 500 & 200 & - & - & 0.950 & 0.92 & 1.05 & 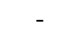 & - & - & - & - & - & - & - \\
\hline 3 & - & - & 700.0 & 69.5 & 1.0 & 0.92 & 1.05 & 150 & 1500 & -500 & 750 & 200 & 0.01 & 25 & 100 \\
\hline 4 & - & - & 600.0 & 304.9 & 1.0 & 0.92 & 1.05 & 150 & 1500 & -500 & 750 & 200 & 0.01 & 60 & 100 \\
\hline 5 & - & - & 333.8 & 146.9 & 1.0 & 0.92 & 1.05 & 150 & 1500 & -500 & 750 & 200 & 0.01 & 30 & 100 \\
\hline
\end{tabular}

$a, b, c$ : Cost coefficients of conventional generators in nonlinear form $a P_{G}^{2}+b P_{G}+c$

TABLE III

5-BUS SYSTEM: LINE DATA.

\begin{tabular}{cccccccc}
\hline & bus & bus & $V^{n o m}$ & $R_{n m}$ & $X_{n m}$ & $B_{n m}$ & $I_{n m}^{\max }$ \\
line & $n$ & $m$ & $\mathrm{kV}$ & $\Omega$ & $\Omega$ & $\mu \mathrm{S}$ & $\mathrm{A}$ \\
\hline L1 & 1 & 2 & 400 & 3.2 & 16 & 160 & 1587.7 \\
L2 & 1 & 3 & 400 & 6.4 & 32 & 320 & 1587.7 \\
L3 & 1 & 4 & 400 & 3.2 & 16 & 160 & 1587.7 \\
L4 & 2 & 5 & 400 & 6.4 & 32 & 320 & 1587.7 \\
L5 & 3 & 4 & 400 & 6.4 & 32 & 320 & 1587.7 \\
L6 & 4 & 5 & 400 & 6.4 & 32 & 320 & 1587.7 \\
\hline
\end{tabular}

TABLE IV

WIND POWER SCENARIOS PROFILE (NORMALIZED VALUES)

\begin{tabular}{ccccccccccc}
\hline$t$ & $\mathrm{~s} 1$ & $\mathrm{~s} 2$ & $\mathrm{~s} 3$ & $\mathrm{~s} 4$ & $\mathrm{~s} 5$ & $\mathrm{~s} 6$ & $\mathrm{~s} 7$ & $\mathrm{~s} 8$ & $\mathrm{~s} 9$ & $\mathrm{~s} 10$ \\
\hline 1 & 0.17 & 0.36 & 0.15 & 0.59 & 0.11 & 0.01 & 0.37 & 0.06 & 0.17 & 0.16 \\
2 & 0.21 & 0.53 & 0.30 & 0.65 & 0.14 & 0.03 & 0.46 & 0.16 & 0.00 & 0.26 \\
3 & 0.07 & 0.26 & 0.24 & 0.59 & 0.26 & 0.09 & 0.09 & 0.12 & 0.02 & 0.11 \\
4 & 0.00 & 0.26 & 0.23 & 0.39 & 0.16 & 0.15 & 0.12 & 0.15 & 0.04 & 0.00 \\
5 & 0.02 & 0.30 & 0.19 & 0.25 & 0.15 & 0.13 & 0.24 & 0.40 & 0.08 & 0.01 \\
6 & 0.00 & 0.19 & 0.30 & 0.06 & 0.08 & 0.17 & 0.29 & 0.58 & 0.08 & 0.01 \\
7 & 0.00 & 0.11 & 0.25 & 0.04 & 0.01 & 0.46 & 0.26 & 0.73 & 0.03 & 0.00 \\
8 & 0.00 & 0.01 & 0.33 & 0.16 & 0.03 & 0.33 & 0.27 & 0.88 & 0.04 & 0.01 \\
9 & 0.10 & 0.01 & 0.22 & 0.25 & 0.00 & 0.27 & 0.41 & 0.90 & 0.05 & 0.15 \\
10 & 0.26 & 0.04 & 0.33 & 0.10 & 0.06 & 0.09 & 0.33 & 0.39 & 0.20 & 0.16 \\
11 & 0.19 & 0.07 & 0.25 & 0.14 & 0.14 & 0.11 & 0.57 & 0.40 & 0.46 & 0.13 \\
12 & 0.08 & 0.15 & 0.07 & 0.23 & 0.27 & 0.15 & 0.28 & 0.96 & 0.24 & 0.04 \\
13 & 0.21 & 0.08 & 0.06 & 0.22 & 0.26 & 0.27 & 0.03 & 0.88 & 0.17 & 0.04 \\
14 & 0.40 & 0.15 & 0.14 & 0.13 & 0.20 & 0.29 & 0.07 & 0.89 & 0.03 & 0.16 \\
15 & 0.30 & 0.04 & 0.41 & 0.10 & 0.29 & 0.11 & 0.09 & 0.53 & 0.01 & 0.09 \\
16 & 0.04 & 0.03 & 0.52 & 0.14 & 0.12 & 0.04 & 0.17 & 0.15 & 0.01 & 0.05 \\
17 & 0.18 & 0.02 & 0.45 & 0.15 & 0.06 & 0.17 & 0.30 & 0.03 & 0.06 & 0.11 \\
18 & 0.21 & 0.03 & 0.36 & 0.10 & 0.01 & 0.12 & 0.26 & 0.00 & 0.08 & 0.29 \\
19 & 0.09 & 0.08 & 0.11 & 0.01 & 0.01 & 0.04 & 0.07 & 0.07 & 0.09 & 0.16 \\
20 & 0.23 & 0.09 & 0.04 & 0.02 & 0.04 & 0.03 & 0.02 & 0.06 & 0.49 & 0.18 \\
21 & 0.28 & 0.03 & 0.01 & 0.10 & 0.01 & 0.03 & 0.02 & 0.03 & 0.30 & 0.04 \\
22 & 0.38 & 0.00 & 0.00 & 0.34 & 0.07 & 0.04 & 0.11 & 0.05 & 0.31 & 0.09 \\
23 & 0.11 & 0.00 & 0.00 & 0.37 & 0.02 & 0.10 & 0.15 & 0.04 & 0.14 & 0.41 \\
24 & 0.10 & 0.02 & 0.04 & 0.27 & 0.01 & 0.06 & 0.48 & 0.13 & 0.10 & 0.89 \\
\hline
\end{tabular}

RC10, Fig. 3 illustrates the state of charge (SoC) profile for scenario 8 (i.e. 88 in Table IV] while Fig. 4 plots and ESS and FL profiles. As expected, to accommodate maximum wind power in the network, ESS discharges in periods with excess of wind (i.e. 7, 8, 9, 12, 13 and 14) while, with the same trend, FL decreases the load (i.e. underdemand) during the same periods. To maintain their daily energy balance equal to

TABLE V

ESS CHARACTERISTICS

\begin{tabular}{ccccccc}
\hline bus & $\begin{array}{c}S O C_{e}^{\min } \\
\mathrm{MWh}\end{array}$ & $\begin{array}{c}S O C_{e}^{\max } \\
\mathrm{MWh}\end{array}$ & $\begin{array}{c}\bar{P}_{e}^{c h} \\
\mathrm{MW}\end{array}$ & $\begin{array}{c}\bar{P}_{e}^{\text {dis }} \\
\mathrm{MW}\end{array}$ & $\eta^{\text {ch,e }}$ & $\eta^{\text {dis }, e}$ \\
\hline 1 & 660 & 2200 & 50 & 50 & 0.95 & 0.95 \\
\hline
\end{tabular}

TABLE VI

CASE\#0 SCOPF RESULTS FOR DIFFERENT RES CAPACITIES

\begin{tabular}{cccccc}
\hline $\begin{array}{c}\text { RES } \\
\text { Cases }\end{array}$ & $\begin{array}{c}\text { RES } \\
(\mathbf{M W})\end{array}$ & $\begin{array}{c}\text { CG } \\
\text { cost }(€)\end{array}$ & $\begin{array}{c}\text { LC } \\
\text { cost }(€)\end{array}$ & $\begin{array}{c}\text { Total } \\
\text { cost }(€)\end{array}$ & $\begin{array}{c}\text { Time } \\
\text { (s) }\end{array}$ \\
\hline RC0 & 0 & $1,693,208$ & 0 & $1,693,208$ & 12 \\
RC1 & 100 & $1,676,410$ & 0 & $1,676,410$ & 11 \\
RC2 & 200 & $1,659,782$ & 0 & $1,659,782$ & 12 \\
RC3 & 300 & $1,643,324$ & 0 & $1,643,324$ & 11 \\
RC4 & 400 & $1,627,036$ & 0 & $1,627,036$ & 12 \\
RC5 & 500 & $1,610,917$ & 0 & $1,610,917$ & 13 \\
RC6 & 600 & $1,594,967$ & 0 & $1,594,967$ & 14 \\
RC7 & 700 & $1,578,949$ & 2,287 & $1,581,236$ & 21 \\
RC8 & 800 & $1,560,524$ & 2,8060 & $1,588,584$ & 25 \\
RC9 & 900 & $1,543,530$ & 57,444 & $1,600,974$ & 25 \\
RC10 & 1,000 & $1,526,703$ & 105,294 & $1,631,997$ & 26 \\
\hline
\end{tabular}

TABLE VII

CASE\#1 SCOPF RESULTS WITH ESS FOR DIFFERENT RES CAPACITIES

\begin{tabular}{cccccc}
\hline $\begin{array}{c}\text { RES } \\
\text { Cases }\end{array}$ & $\begin{array}{c}\text { CG Cost } \\
(€)\end{array}$ & $\begin{array}{c}\text { LC cost } \\
(€)\end{array}$ & $\begin{array}{c}\text { ESS cost } \\
(€)\end{array}$ & $\begin{array}{c}\text { Total cost } \\
(€)\end{array}$ & $\begin{array}{c}\text { Time } \\
(\mathbf{s})\end{array}$ \\
\hline RC0 & $1,693,208$ & 0 & 0 & $1,693,208$ & 12 \\
RC1 & $1,676,410$ & 0 & 0 & $1,676,410$ & 13 \\
RC2 & $1,659,782$ & 0 & 0 & $1,659,782$ & 13 \\
RC3 & $1,643,324$ & 0 & 0 & $1,643,324$ & 13 \\
RC4 & $1,627,036$ & 0 & 0 & $1,627,036$ & 13 \\
RC5 & $1,610,917$ & 0 & 0 & $1,610,917$ & 14 \\
RC6 & $1,594,967$ & 0 & 0 & $1,594,967$ & 16 \\
RC7 & $1,579,193$ & 0 & 389 & $1,579,582$ & 23 \\
RC8 & $1,563,451$ & 2,064 & 4,390 & $1,569,905$ & 27 \\
RC9 & $1,546,819$ & 26,052 & 5,300 & $1,578,171$ & 32 \\
RC10 & $1,530,821$ & 56,754 & 8,332 & $1,595,907$ & 27 \\
\hline
\end{tabular}

zero, both ESS and FL increase charging and load to hours of low wind (i.e. 1-6 and 16-24).

Fig. 5 illustrates the load curtailment for scenario s8 and contingency in line L2. A decreasing trend can be observed from Case\#0 to Case\#3 by considering the flexibility of ESS and FL. For instance, at $12 \mathrm{pm}$, the curtailed power reduces by $72 \%$ (i.e. $(1.714-0.42) / 1.714)$ when considering both ESS and FL. This further demonstrates the benefits of additional flexibility offered by ESS and FL.

\section{B. Results for Nordic32 Test System}

To test model scalability we use the synthetic Nordic32 test system [39], which is closely inspired by the Sweden system. The test system includes 60 nodes, 23 generators, 57 lines, 31 transformers, and 12 shunts reactors/capacitors [39]. A contingency set of $33 \mathrm{~N}-1$ line disconnections is assumed. We assume a futuristic renewable-dominated version of this 
TABLE VIII

CASE\#2 SCOPF RESULTS WITH FL FOR DIFFERENT RES CAPACITIES

\begin{tabular}{cccccc}
\hline $\begin{array}{c}\text { RES } \\
\text { Cases }\end{array}$ & $\begin{array}{c}\text { CG Cost } \\
(€)\end{array}$ & $\begin{array}{c}\text { LC cost } \\
(€)\end{array}$ & $\begin{array}{c}\text { FL cost } \\
(€)\end{array}$ & $\begin{array}{c}\text { Total cost } \\
(€)\end{array}$ & $\begin{array}{c}\text { Time } \\
(\mathbf{s})\end{array}$ \\
\hline RC0 & $1,693,208$ & 0 & 0 & $1,693,208$ & 12 \\
RC1 & $1,676,410$ & 0 & 0 & $1,676,410$ & 13 \\
RC2 & $1,659,782$ & 0 & 0 & $1,659,782$ & 13 \\
RC3 & $1,643,324$ & 0 & 0 & $1,643,324$ & 13 \\
RC4 & $1,627,036$ & 0 & 0 & $1,627,036$ & 13 \\
RC5 & $1,610,917$ & 0 & 0 & $1,610,917$ & 13 \\
RC6 & $1,594,967$ & 0 & 0 & $1,594,967$ & 14 \\
RC7 & $1,579,165$ & 0 & 550 & $1,579,715$ & 26 \\
RC8 & $1,563,507$ & 0 & 6,710 & $1,570,217$ & 20 \\
RC9 & $1,548,656$ & 11,780 & 10,899 & $1,571,335$ & 29 \\
RC10 & $1,532,739$ & 40,001 & 14,377 & $1,587,117$ & 33 \\
\hline
\end{tabular}

TABLE IX

CASE\#3 SCOPF RESULTS WITH ESS AND FL FOR 10 RES CAPACITIES

\begin{tabular}{ccccccc}
\hline $\begin{array}{c}\text { RES } \\
\text { Cases }\end{array}$ & $\begin{array}{c}\text { CG } \\
\text { Cost }(\boldsymbol{\epsilon})\end{array}$ & $\begin{array}{c}\text { LC } \\
\text { Cost }(\boldsymbol{\epsilon})\end{array}$ & $\begin{array}{c}\text { FL } \\
\text { Cost }(\boldsymbol{\xi})\end{array}$ & $\begin{array}{c}\text { ESS } \\
\text { Cost }(\boldsymbol{\epsilon})\end{array}$ & $\begin{array}{c}\text { Total } \\
\text { Cost }(\boldsymbol{\epsilon})\end{array}$ & $\begin{array}{c}\text { Time } \\
(\mathbf{s})\end{array}$ \\
\hline RC0 & $1,693,208$ & 0 & 0 & 0 & $1,693,208$ & 14 \\
RC1 & $1,676,410$ & 0 & 0 & 0 & $1,676,410$ & 14 \\
RC2 & $1,659,782$ & 0 & 0 & 0 & $1,659,782$ & 14 \\
RC3 & $1,643,324$ & 0 & 0 & 0 & $1,643,324$ & 14 \\
RC4 & $1,627,036$ & 0 & 0 & 0 & $1,627,036$ & 14 \\
RC5 & $1,610,917$ & 0 & 0 & 0 & $1,610,917$ & 14 \\
RC6 & $1,594,967$ & 0 & 0 & 0 & $1,594,967$ & 15 \\
RC7 & $1,579,193$ & 0 & 0 & 389 & $1,579,582$ & 26 \\
RC8 & $1,563,707$ & 0 & 491 & 4,390 & $1,568,588$ & 24 \\
RC9 & $1,550,080$ & 0 & 6200 & 5,299 & $1,561,579$ & 23 \\
RC10 & $1,536,615$ & 10,001 & 12,956 & 6,065 & $1,565,637$ & 30 \\
\hline
\end{tabular}

system (see Fig. 6), in which five large wind farms (with $7,200,5,400,6,300,5,700$, and 6,300 MW of rated power) are installed at nodes 1012, 1013, 1014, 4021 and 4042, respectively. As a consequence, to cope with the uncertain variability and potential excess of active power injected in north area while managing congestion and voltage issues, three FL are assumed at nodes 1011, 1044, and 2031, and two ESS

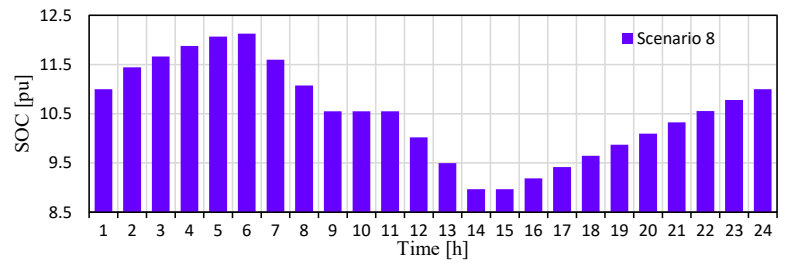

Fig. 3. SOC profile of ESS for scenario 8 in contingency in line L2

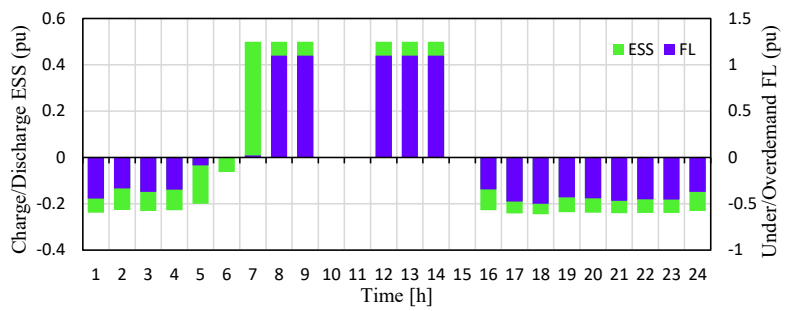

Fig. 4. Injection and absorption profile of active power for both ESS and FL in contingency in line L2

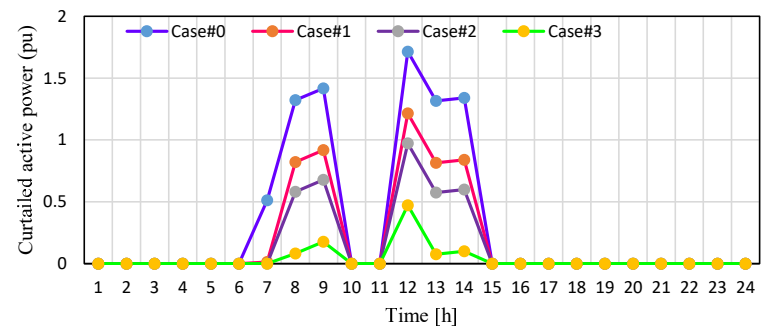

Fig. 5. Curtailed active power profile for four cases: contingency L2 and s8

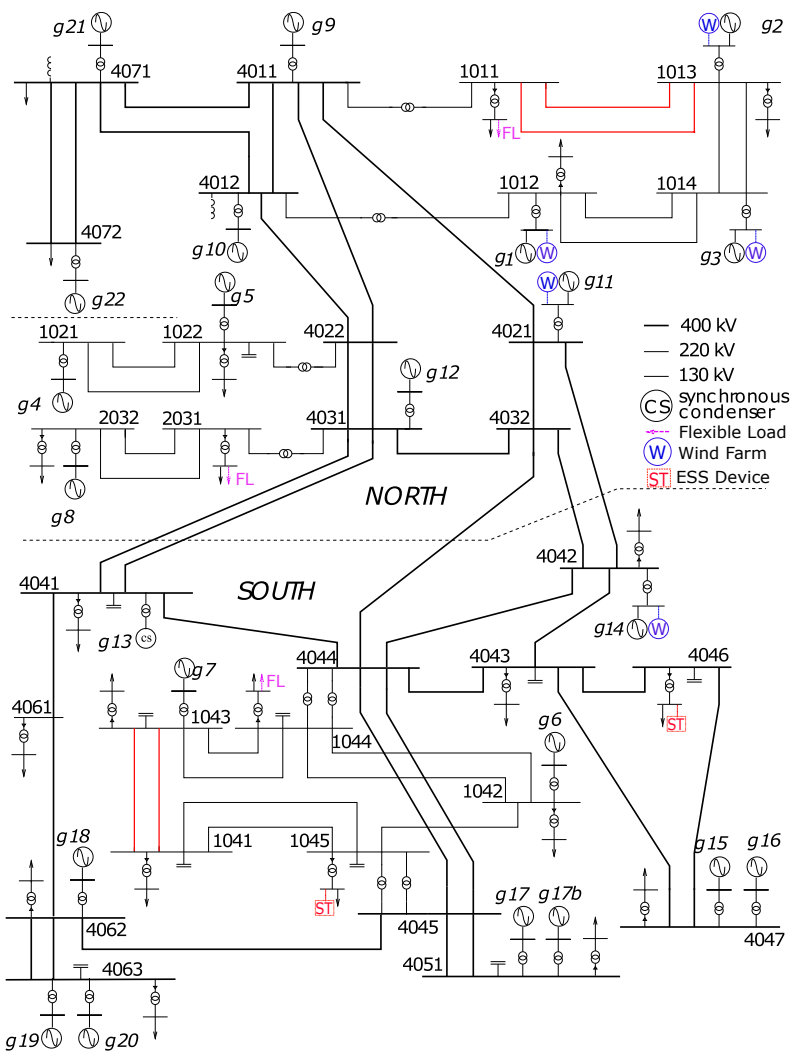

Fig. 6. One-line diagram of Nordic32 test system

(with the same parameters as in Table $\mathrm{V}$ ) are embedded at nodes 1045 and 4046. The load pattern from [40] is adopted for a generic summer day. To test the scalability of the proposed model, we consider up to 30 scenarios.

1) Illustration of flexibility resources: To evaluate the added value of using emerging flexible resources (FL and ESS), like for the 5-bus system, four different case studies are defined, namely: Case\#0 (base case) in which neither FL nor ESS are used, Case\#1 where only ESS units are utilized, Case\#2 where only FL are considered, and finally, Case\#3 where both ESS and FL units are available. For all cases, 10 wind scenarios are generated, the ESS cost is set to $3 € / M W h$ and FL cost is set to $2.5 € / \mathrm{MWh}$ for both normal operation and postcontingency states. The load and generation curtailment cost is set to $30 € / M W h$ i.e. ten times larger than the most expensive conventional generator cost. 
TABLE $X$

NORDIC32 TEST SYSTEM RESULTS FOR DIFFERENT CASE STUDIES

\begin{tabular}{|c|c|c|c|c|c|c|c|c|c|c|}
\hline \multirow[b]{2}{*}{ Cases } & \multicolumn{5}{|c|}{ normal operation state } & \multicolumn{4}{|c|}{ post contingency state } & \multirow[b]{2}{*}{$\begin{array}{c}\text { Total } \\
\text { cost }(€)\end{array}$} \\
\hline & $\begin{array}{c}C G \\
\operatorname{cost}(€)\end{array}$ & $\begin{array}{c}\mathrm{LC} \\
\operatorname{cost}(€)\end{array}$ & $\begin{array}{c}\text { GC } \\
\operatorname{cost}(€)\end{array}$ & $\begin{array}{c}\mathrm{FL} \\
\operatorname{cost}(€)\end{array}$ & $\begin{array}{c}\text { ESS } \\
\operatorname{cost}(€)\end{array}$ & $\begin{array}{c}\mathrm{LC} \\
\operatorname{cost}(€)\end{array}$ & $\begin{array}{c}\text { GC } \\
\operatorname{cost}(€)\end{array}$ & $\begin{array}{c}\mathrm{FL} \\
\operatorname{cost}(€)\end{array}$ & $\begin{array}{c}\text { ESS } \\
\operatorname{cost}(€)\end{array}$ & \\
\hline Case\#0 & 212,184 & 0.0 & 16,726 & - & -1 & 1,791 & 26,754 & - & -1 & 257,456 \\
\hline Case\#1 & 211,688 & 0.0 & 16,726 & - & 0.0 & 1,791 & 26,758 & - & 382 & 257,344 \\
\hline Case\#2 & 212,161 & 0.0 & 16,153 & 226 & - & 1,791 & 25,873 & 364 & - & 256,571 \\
\hline Case\#3 & 211,675 & 0.0 & 16,158 & 226 & 0.0 & 1,791 & 25,880 & 361 & 369 & 256,464 \\
\hline
\end{tabular}

Table $\mathrm{X}$ provides the different components of the total expected cost for the different cases. In case\#0 the total cost equals $257,456 €$ and wind generation curtailment occurs in both normal and post contingency states. However, thanks to the additional flexibility offered by the ESS in node 1045, the conventional operation cost reduces from $212,184 €$ in the Case\#0 to $211,688 €$ in the Case\#1. As a result, although an additional cost regarding the activation of ESS is imposed in the post contingency state, total cost decreases from $257,456 €$ to $257,344 €$. In Case\#2, although the conventional generators' cost remains almost constant, the wind generation curtailment cost is reduced meaningfully, from $26,754 €$ in the base case to $25,873 €$ in Case\#2. Consequently, the total expected cost reduces by $885 €$ (i.e. $257,456-256,571=885 €$ ) as compared to the base case. In addition, in Case\#2, the wind power curtailment cost in normal operation is also reduced by $573 €$ (i.e. $16,726-16,153=573 €$ ). The same trend can be observed in the last case, Case\#4, where both ESS and FL are activated in the post contingency state, where the additional volume of flexibility causes a total expected cost reduction of $992 €$ (i.e. from $257,456 €$ in Case\#0 to $256,464 €$ in Case\#3).

These results demonstrate that flexible resources (ESS and FL) can contribute cost-effectively to a reduction of wind energy spillage and load curtailment, improving the overall system flexibility, and allowing thereby to accommodate larger amounts of renewables.

Figs. 7 and 8 illustrate the flexible resources behaviour at the solution of the proposed S-MP-SCOPF model. Fig. 7 shows the SoC profile of ESS at node 1045 in scenario s1 and the contingency in a single line circuit between nodes 1041 and 1043, which overloads the second line circuit in parallel between the same nodes, both colored in red in Fig. 6. As expected, to alleviate the overload in the second circuit of line 1041-1043 in periods with high demand, ESS discharges during peak hours (i.e. 17-20) and charges during the lower demand hours where the line is less loaded to maintain its energy balance constraint (see Fig. 7).

The FL at node 1011 in the same scenario and contingency shows apparently a counter-intuitive behavior. As can be seen in Fig. 8, FL decreases the load during hours with extra wind power generation and, to maintain its daily energy balance, increases the load in hours with high load. This behaviour can be justified as follows. In hours 7-9 and 12-14, when wind farms generate large amount of wind power, the lines between nodes 1011 and 1013, shown with red colour in Fig. 6 are congested in both normal and post-contingency states.

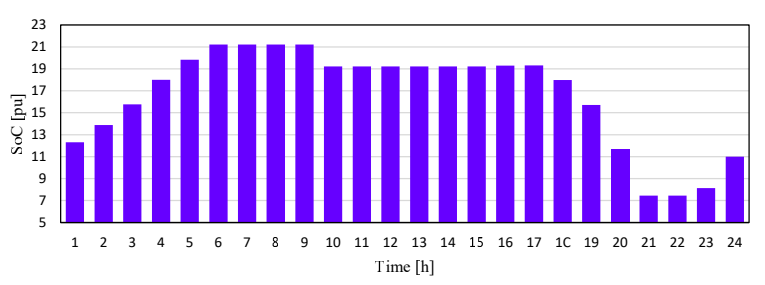

Fig. 7. SOC profile of ESS at node 1045 in scenario s1 and contingency in the connecting line between nodes 1041 and 1043

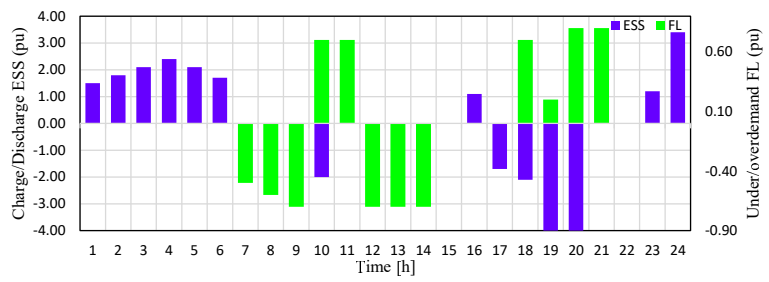

Fig. 8. Injection and absorption profile of active power for both ESS and FL and contingency in the connecting line between nodes 1041 and 1043

These bottlenecks require the activation of FL to remove these congestions (by creating counter-flows) and minimize the wind power curtailment. In conclusion, the primary functionality of FL is driven by the prevention of current/voltage constraints violation rather than the simpler power balance satisfaction needs.

2) Model scalability: Table XI shows the results of the scalability test of the proposed S-MP-SCOPF model for increasing number of scenarios and thereby problem size. The results are obtained with IPOPT solver using default setting except of the relative optimality gap tolerance, which is set to $10^{-5}$.

Note that while increasing the problem size, the elapsed time grows sharply with non-monotonic slope. For instance, although the problem size increases ten times from 1 scenario to 10 scenarios, the computation time increases more than 19.7 times (i.e. $(5,985-289) / 289)$. In addition, the largest number of scenarios the solver can handle reliably is 30 , which corresponds to a huge NLP optimization problem with roughly 5 millions continues variables and 9 millions of constraints, which is solved in 22,110 seconds. For larger number of scenarios the default linear (system of equations) solver package MUMPS in IPOPT fails to allocate memory even before IPOPT can start iterations. An interesting observation regarding IPOPT solver is that, as for other interior-point method-based solvers, the iteration number is little dependent 
TABLE XI

NORDIC32 TEST SYSTEM RESULTS FOR DIFFERENT SCENARIOS

\begin{tabular}{cccccc}
\hline $\begin{array}{c}\text { Number of } \\
\text { scenarios }\end{array}$ & $\begin{array}{c}\text { Total } \\
\text { cost }(\boldsymbol{€})\end{array}$ & $\begin{array}{c}\text { Continues } \\
\text { variables }\end{array}$ & Constraints & Iter & $\begin{array}{c}\text { Time } \\
(\mathbf{s})\end{array}$ \\
\hline 1 & 555,874 & 197,206 & 287,596 & 123 & 289 \\
2 & 421,924 & 394,582 & 575,192 & 144 & 764 \\
3 & 652,595 & 591,788 & 862,788 & 167 & 1,404 \\
4 & 319,122 & 789,164 & $1,150,384$ & 173 & 2,040 \\
5 & 297,430 & 986,540 & $1,437,980$ & 160 & 2,283 \\
6 & 284,137 & $1,183,916$ & $1,725,576$ & 166 & 2,878 \\
7 & 274,769 & $1,381,292$ & $2,013,172$ & 171 & 3,596 \\
8 & 267,411 & $1,578,668$ & $2,300,768$ & 169 & 4,100 \\
9 & 261,006 & $1,776,044$ & $2,588,364$ & 180 & 4,451 \\
10 & 256,464 & $1,973,420$ & $2,875,960$ & 186 & 5,985 \\
20 & 256,464 & $3,312,280$ & $5,751,920$ & 185 & 14,351 \\
30 & 256,464 & $4,968,420$ & $8,627,880$ & 186 & 22,110 \\
40 & IPOPT failed & & & & \\
\hline
\end{tabular}

on the size of the problem.

Note that the computation time can be further significantly improved by using another linear solver in IPOPT. For example, it is reported at https://github.com/power-grid-lib/pglibopf/blob/master/BASELINE.md that ma27 linear solver can decrease the runtime by 2-6 times as compared to default linear solver MUMPS. However, we did not manage to compile and plug ma27 linear solver in our windows code implemenation to test its performances.

Finally, in terms of objective function one can observe that, since in cases with 1 and 2 scenarios large amount of wind power is injected into the system, generation curtailment occurs in peak hours which causes an increase in total cost for these two cases. Note that we initially generated 10 scenarios as in Table IV] and for the cases with larger numbers, the scenarios are replicated out of the original set of scenarios. For this reason the value of total cost remains unchanged for the cases with more than 10 scenarios.

\section{CONCLUSiOnS AND FUtURE WORK}

The research efforts devoted to address the challenge of extending the state-of-the-art in AC SCOPF (i.e. deterministic and single time period) is scarce and mostly capture one novel feature at the time. This paper has extended the state-ofthe-art in AC SCOPF to capture two new dimensions (RES stochasticity and multiple time periods) as well as to model time dependent constraints of emerging sources of flexibility (FL and ESS). Accordingly, this paper solves for the first time a new NLP problem formulation in the form of stochastic multi-period AC SCOPF (S-MP-SCOPF) which we envision for procuring flexibility for ancillary services (congestion and voltage control) in renewable supply dominated power systems of the future. This problem enables computing optimal set points of the flexibility resources and other conventional control means for congestion management and voltage control in day-ahead operation planning.

As we address a new problem, full problem details and results have been provided for a 5 -node test system to foster benchmarking. The results obtained for this system show the effectiveness of the ESS and FL for flexibility provision in day-ahead operation, which are able to reduce the load curtailment cost up to $90.5 \%$.

The Nordic32 test system has been used to ascertain scalability, noting that as shown in Table I scalability is rarely addressed by the few works that extend the state-of-the-art in AC SCOPF. The largest NLP S-MP-SCOPF problem solved (60 nodes, 34 states, 24 time periods, 30 scenarios) is roughly equivalent in size to solving an AC OPF problem for a system of huge size (cca. 1,500,000 nodes). Very few works report results for such a big NLP problem. We have relied on the state-of-the-art NLP solver IPOPT, which is widely used to solve AC OPF/SCOPF problems. The running time obtained for the largest NLP problem on this system (roughly 5 millions optimisation variables and 9 millions constraints, which is very close to the edge of computer/solver limit) is 22,110 seconds. While this time could be deemed a bit excessive for dayahead operation planning, the elapsed time for a problem that includes three times less scenarios is 5,985 seconds, which is still acceptable.

The paper has discussed that a massive reduction in computation time could be expected by using a more performant linear solver within IPOPT, a tailored implementation parallelizing some computations [11], merely using a commercial solver, or developing iterative methodologies [9].

This direct approach can thus scale to medium size systems by careful beforehand knowledge of problematic/binding contingencies, as an input from the operator, as well as reducing the number of uncertainty scenarios to a few.

As future work, we plan to develop a tractable approach of S-MP-SCOPF problem through decomposition and approximation along the above mentioned lines.

\section{REFERENCES}

[1] N. Balu, T. Bertram, A. Bose, V. Brandwajn, G. Cauley, D. Curtice, A. Fouad, L. Fink, M. G. Lauby, B. F. Wollenberg, et al., "On-line power system security analysis," Proceedings of the IEEE, vol. 80, no. 2, pp. 262-282, 1992.

[2] M. Alizadeh, M. P. Moghaddam, N. Amjady, P. Siano, and M. SheikhEl-Eslami, "Flexibility in future power systems with high renewable penetration: A review," Renewable and Sustainable Energy Reviews, vol. 57, pp. 1186-1193, 2016.

[3] Q. Wang and B.-M. Hodge, "Enhancing power system operational flexibility with flexible ramping products: A review," IEEE Transactions on Industrial Informatics, vol. 13, no. 4, pp. 1652-1664, 2016.

[4] F. Capitanescu, J. M. Ramos, P. Panciatici, D. Kirschen, A. M. Marcolini, L. Platbrood, and L. Wehenkel, "State-of-the-art, challenges, and future trends in security constrained optimal power flow," Electric Power Systems Research, vol. 81, no. 8, pp. 1731-1741, 2011.

[5] B. Stott and O. Alsaç, "Optimal power flow: Basic requirements for real-life problems and their solutions," in SEPOPE XII Symposium, Rio de Janeiro, Brazil, vol. 11, 2012.

[6] F. Capitanescu, "Challenges ahead risk-based ac optimal power flow under uncertainty for smart sustainable power systems," Dynamic Vulnerability Assessment and Intelligent Control: For Sustainable Power Systems, p. 149, 2018.

[7] F. Capitanescu, M. Glavic, D. Ernst, and L. Wehenkel, "Applications of security-constrained optimal power flows," in In Proceedings of Modern Electric Power Systems Symposium, MEPS06, 2006.

[8] D. Phan and J. Kalagnanam, "Some efficient optimization methods for solving the security-constrained optimal power flow problem," IEEE Transactions on Power Systems, vol. 29, no. 2, pp. 863-872, 2013. 
[9] L. Platbrood, F. Capitanescu, C. Merckx, H. Crisciu, and L. Wehenkel, "A generic approach for solving nonlinear-discrete security-constrained optimal power flow problems in large-scale systems," IEEE Transactions on Power Systems, vol. 29, no. 3, pp. 1194-1203, 2013.

[10] Q. Jiang and $\mathrm{K} . \mathrm{Xu}$, "A novel iterative contingency filtering approach to corrective security-constrained optimal power flow," IEEE Transactions on Power Systems, vol. 29, no. 3, pp. 1099-1109, 2013.

[11] J. Kardoš, D. Kourounis, and O. Schenk, “Two-level parallel augmented schur complement interior-point algorithms for the solution of security constrained optimal power flow problems," IEEE Transactions on Power Systems, vol. 35, no. 2, pp. 1340-1350, 2019.

[12] I.-I. Avramidis, F. Capitanescu, S. Karagiannopoulos, and E. Vrettos, "A novel approximation of security-constrained optimal power flow with incorporation of generator frequency and voltage control response," IEEE Transactions on Power Systems, 2020.

[13] L. de Magalhães Carvalho, A. M. L. da Silva, and V. Miranda, "Security-constrained optimal power flow via cross-entropy method," IEEE Transactions on Power Systems, vol. 33, no. 6, pp. 6621-6629, 2018.

[14] R. Madani, M. Ashraphijuo, and J. Lavaei, "Promises of conic relaxation for contingency-constrained optimal power flow problem," IEEE Transactions on Power Systems, vol. 31, no. 2, pp. 1297-1307, 2015.

[15] M. Yan, M. Shahidehpour, A. Paaso, C. Zhang, A. Abdulwhab, and A. Abusorrah, "A convex three-stage scopf approach to power system flexibility with unified power flow controllers," IEEE Transactions on Power Systems, pp. 1-1, 2020.

[16] T. Valencia-Zuluaga, D. Agudelo-Martinez, D. Arango-Angarita, C. Acosta-Urrego, S. Rivera, D. Rodríguez-Medina, and J. Gers, "A fast decomposition method to solve a security-constrained optimal power flow (scopf) problem through constraint handling," IEEE Access, vol. 9, pp. 52812-52824, 2021.

[17] A. Velloso, P. Van Hentenryck, and E. S. Johnson, "An exact and scalable problem decomposition for security-constrained optimal power flow," Electric Power Systems Research, vol. 195, p. 106677, 2021.

[18] R. Weinhold and R. Mieth, "Fast security-constrained optimal power flow through low-impact and redundancy screening," IEEE Transactions on Power Systems, vol. 35, no. 6, pp. 4574-4584, 2020.

[19] M. Velay, M. Vinyals, Y. Besanger, and N. Retière, "Fully distributed security constrained optimal power flow with primary frequency control," International Journal of Electrical Power \& Energy Systems, vol. 110, pp. 536-547, 2019.

[20] F. Karbalaei, H. Shahbazi, and M. Mahdavi, "A new method for solving preventive security-constrained optimal power flow based on linear network compression," International Journal of Electrical Power \& Energy Systems, vol. 96, pp. 23-29, 2018.

[21] A. Velloso and P. Van Hentenryck, "Combining deep learning and optimization for preventive security-constrained dc optimal power flow," IEEE Transactions on Power Systems, 2021.

[22] F. Capitanescu, S. Fliscounakis, P. Panciatici, and L. Wehenkel, "Cautious operation planning under uncertainties," IEEE Transactions on Power Systems, vol. 27, no. 4, pp. 1859-1869, 2012.

[23] L. You, H. Ma, T. Saha, and G. Liu, "Risk-based contingencyconstrained optimal power flow with adjustable uncertainty set of wind power," IEEE Transactions on Industrial Informatics, 2021.

[24] M. Vrakopoulou, M. Katsampani, K. Margellos, J. Lygeros, and G. Andersson, "Probabilistic security-constrained ac optimal power flow," in 2013 IEEE Grenoble Conference, pp. 1-6, IEEE, 2013.

[25] E. Nycander and L. Söder, "Comparison of stochastic and deterministic security constrained optimal power flow under varying outage probabilities," in 2019 IEEE Milan PowerTech, pp. 1-6, 2019.

[26] A. Venzke and S. Chatzivasileiadis, "Convex relaxations of security constrained ac optimal power flow under uncertainty," in 2018 Power Systems Computation Conference (PSCC), pp. 1-7, IEEE, 2018.

[27] C. Hamon, M. Perninge, and L. Söder, "The value of using chanceconstrained optimal power flows for generation re-dispatch under uncertainty with detailed security constraints," in 2013 IEEE PES Asia-Pacific Power and Energy Engineering Conference (APPEEC), pp. 1-6, IEEE, 2013.

[28] E. Karangelos and L. Wehenkel, "An iterative ac-scopf approach managing the contingency and corrective control failure uncertainties with a probabilistic guarantee," IEEE Transactions on Power Systems, vol. 34, no. 5, pp. 3780-3790, 2019.

[29] H. Sharifzadeh, N. Amjady, and H. Zareipour, "Multi-period stochastic security-constrained opf considering the uncertainty sources of wind power, load demand and equipment unavailability," Electric Power Systems Research, vol. 146, pp. 33-42, 2017.

[30] C. E. Murillo-Sánchez, R. D. Zimmerman, C. L. Anderson, and R. J. Thomas, "Secure planning and operations of systems with stochastic sources, energy storage, and active demand," IEEE Transactions on Smart Grid, vol. 4, no. 4, pp. 2220-2229, 2013.

[31] A. Fuchs, J. Garrison, and T. Demiray, "A security-constrained multiperiod opf for the locational allocation of automatic reserves," in 2017 IEEE Manchester PowerTech, pp. 1-6, IEEE, 2017.

[32] M. Schanen, F. Gilbert, C. G. Petra, and M. Anitescu, "Toward multiperiod ac-based contingency constrained optimal power flow at large scale," in 2018 Power Systems Computation Conference (PSCC), pp. 17, IEEE, 2018.

[33] J. Cao, W. Du, and H. Wang, "An improved corrective security constrained opf with distributed energy storage," IEEE Transactions on Power Systems, vol. 31, no. 2, pp. 1537-1545, 2015.

[34] A. Wächter, L. Biegler, Y. Lang, and A. Raghunathan, "Ipopt: An interior point algorithm for large-scale nonlinear optimization," 2002.

[35] D. Gayme and U. Topcu, "Optimal power flow with large-scale storage integration," IEEE Transactions on Power Systems, vol. 28, no. 2, pp. 709-717, 2012.

[36] Z. Shen, W. Wei, D. Wu, T. Ding, and S. Mei, "Modeling arbitrage of an energy storage unit without binary variables," CSEE Journal of Power and Energy Systems, vol. 7, no. 1, pp. 156-161, 2020.

[37] I. Dunning, J. Huchette, and M. Lubin, "Jump: A modeling language for mathematical optimization," SIAM review, vol. 59, no. 2, pp. 295-320, 2017.

[38] K. C. Sharma, P. Jain, and R. Bhakar, "Wind power scenario generation and reduction in stochastic programming framework," Electric Power Components and Systems, vol. 41, no. 3, pp. 271-285, 2013.

[39] T. Van Cutsem and L. Papangelis, "Description, modeling and simulation results of a test system for voltage stability analysis," tech. rep., université de Liège, 2013.

[40] I. Pena, C. B. Martinez-Anido, and B.-M. Hodge, "An extended ieee 118bus test system with high renewable penetration," IEEE Transactions on Power Systems, vol. 33, no. 1, pp. 281-289, 2017. 